\title{
Numerical Integrators for Stiff and Highly Oscillatory Differential Equations
}

\author{
By Simeon Ola Fatunla*
}

\begin{abstract}
Some $L$-stable fourth-order explicit one-step numerical integration formulas which require no matrix inversion are proposed to cope effectively with systems of ordinary differential equations with large Lipschitz constants (including those having highly oscillatory solutions). The implicit integration procedure proposed in Fatunla [11] is further developed to handle a larger class of stiff systems as well as those with highly oscillatory solutions. The same pair of nonlinear equations as in [11] is solved for the stiffness/oscillatory parameters. However, the nonlinear systems are transformed into linear forms and an efficient computational procedure is developed to obtain these parameters. The new schemes compare favorably with the backward differentiation formula (DIFSUB) of Gear [13], [14] and the blended linear multistep methods of Skeel and Kong [24], and the symmetric multistep methods of Lambert and Watson [17].
\end{abstract}

1. Introduction. The development of numerical integration formulas for stiff as well as highly oscillatory systems of differential equations has attracted considerable attention in the past decade. The reason for this cannot be farfetched, realizing that the mathematical models of physical situations in kinetic chemical reactions, process control and electrical circuit theory often generate systems of ordinary differential equations whose Jacobians have at least one eigenvalue with a very negative real part or very large imaginary part. These two situations are, respectively, described as stiff and highly oscillatory.

Consider the following model problems

$$
y^{\prime}=\lambda(y-c(x))+c^{\prime}(x), \quad y(a)=y_{0},
$$

where $y(x) \in R^{1}, \lambda$ a complex constant with re $\lambda<<0$ and $c(x)$ is slowly varying in the finite interval $a \leqslant x \leqslant b$;

$$
z^{\prime}=\left(\begin{array}{cc}
-\xi & \omega \\
-\omega & -\xi
\end{array}\right) z, \quad z(a)=z_{0},
$$

with $z(x) \in R^{2}$ and $\omega>>0, \xi$ a positive constant such that $\xi \approx 0$.

Problem (1.1) has theoretical solution

$$
y(x)=c(x)+y_{0} e^{\lambda x},
$$

Received January 6, 1978; revised July 7, 1978 and March 22, 1979.

AMS (MOS) subject classifications (1970). Primary 65L05; Secondary 65D 30.

Key words and phrases. Stiffness and oscillatory parameters, ordinary differential equations, $L$-stable, eigenvalues, meshsize, explicit, implicit, $A$-stable, exponential fitting.

* Research work was carried out during a summer visit to the Department of Computer Science, University of Illinois at Urbana-Champaign. Work supported in part by the U. S. Energy Research and Development Administration under contract US ERDA/EY-76-S-02-2383. 
whose component $c(x)$ is slowly varying in the specified interval while the second component decays rapidly in the transient phase.

The analytic solution to problem (1.2) is given by

$$
z(x)=e^{-\xi x}\left(\begin{array}{c}
\alpha \cos \omega x+\beta \sin \omega x \\
\beta \cos \omega x-\alpha \sin \omega x
\end{array}\right),
$$

where $\alpha$ and $\beta$ are the arbitrary constants of integration.

The transitory phase for problem (1.1) is of the order of $-1 / \lambda$ while that of problem (1.2) is the entire interval of integration with $\omega / 2 \pi$ complete oscillations per unit interval.

Almost invariably, most conventional numerical integration solvers cannot effectively cope with problems (1.1) and (1.2) as they lack adequate stability characteristics. Any attempt to impose the stability properties will in effect constrain the integration mesh-size to be intolerably small. This may ultimately have adverse effects on the accuracy due to accumulation of roundoff errors. Besides, the computing time and cost may be too excessive.

Existing algorithms developed for problems of the type (1.1) can be classified into the following categories:

(i) generalized Runge-Kutta schemes-Lawson [18],

(ii) implicit Runge-Kutta scheme-Butcher [5],

(iii) trapezoidal rule with extrapolation-Lindberg [19],

(iv) multiderivative multistep formulas-Enright [7],

(v) backward differentiation multistep formulas-Gear [13], [14].

As of now, the most widely used numerical integration code for stiff systems is the class (v) schemes, particularly Gear's DIFSUB [13], [14]. The method is efficient and reliable provided the eigenvalues of the Jacobian are not close to the imaginary axis where the higher-order schemes exhibit poor stability properties (as evidenced from Example 2). Dahlquist [6] established that neither an explicit linear multistep scheme of any order nor an implicit multistep method of order greater than two can be $A$-stable. He proved that the trapezoidal rule (which of course is not $L$-stable as $y_{t} / y_{t-1} \rightarrow 1$ as $\left.\lambda h \rightarrow-\infty\right)$ has the smallest error $( \pm)(1 / 12) h^{3} y\left(\begin{array}{l}3 \\ (x)\end{array}\right.$. Other schemes which behave better than DIFSUB when the eigenvalues are close to the imaginary axis includes the second derivative multistep method (Enright [7]).

As regards problems of the type (1.2), earlier efforts include Gautschi's [12] nonlinear multistep schemes which produce exact solution to algebraic (or trigonometric) polynomials up to certain degrees. The main drawback of this scheme is that it requires an a priori knowledge of the period of the systems under consideration. Other numerical integration solvers for oscillatory systems include Amdursky and Ziv [1][3], Snider and Flemming [25], Miranker and Wahba [21], Miranker et al. [22], Miranker and Veldhuizen [23], and Fatunla [9], [10]. Unfortunately, none of these existing routines has been properly (adequately) put to test for various kinds of oscillatory problems. 
In this paper, we propose some two-point numerical integration formulas which effectively cope with systems of ODEs whose characteristics are identical to problems (1.1) and (1.2).

We shall consider initial value problems

$$
y^{\prime}=f(x, y), \quad y(0)=y_{0},
$$

with $y(x) \in R^{m}$ in the finite interval $S=\left[0, x_{f}\right] \subset R^{1}$, where $x_{f}=N h$ for some positive integer $N>0$. It is assumed that $y(x)$ is sufficiently differentiable. We adopt the vector notation: $y=\left({ }^{1} y,{ }^{2} y, \ldots,{ }^{m} y\right)^{T}, f=\left({ }^{1} f,{ }^{2} f, \ldots,{ }^{m} f\right)^{T}$. The numerical estimates $y_{n}$ to the theoretical solution $y\left(x_{n}\right)$ at the points $x_{n}=n h, n=0(1) N$ are to be generated.

On every subinterval $\left[x_{n}, x_{n}+h\right]$, the theoretical solution $y(x)$ is approximated by either the interpolating function

$$
\widetilde{F}(x)=\left(I-e^{\Omega_{1} x}\right) A-\left(I-e^{-\Omega_{2} x}\right) B+C,
$$

$A, B$, and $C$ being $m$-tuples with real entries, $I$ is the identity matrix, whilst $\Omega_{1}$ and $\Omega_{2}$ are diagonal (stiffness/oscillatory) matrices; or

$$
\widetilde{F}(x)=\left(I-e^{\Omega_{1} x}\right) A+\left(I-e^{\Omega_{1}^{*} x}\right) A^{*}+B,
$$

where $A, B$ are $m$-tuples with complex entries and $\left({ }^{*}\right)$ denotes complex conjugate.

The choice of interpolation formula is determined by Eq. (3.15).

The following definitions are worthwhile:

Definition 1. A one-step numerical integration scheme is considered $L$-stable if apart from being $A$-stable, when it is applied to the scalar initial value problem

$$
y^{\prime}=\lambda y, \quad y(0)=\eta
$$

( $\lambda$ being a complex constant with negative real part), the resultant numerical solution is given by

$$
y_{n+1}=\mu(\lambda h) y_{n},
$$

with the characteristic equation $\mu(\lambda h)$ having the property:

$$
\lim _{r e(\lambda h) \rightarrow-\infty}|\mu(\lambda h)|=0 .
$$

Definition 2. A numerical integration scheme is said to be exponentially fitted at a complex value $\lambda=\lambda_{0}$ if when it is applied to the initial value problem (1.8) with exact initial condition, the characteristic equation $\mu(\lambda h)$ satisfies the relation

$$
\mu\left(\lambda_{0} h\right)=e^{\lambda_{0} h}
$$

Liniger and Willoughby [20] and Jackson and Kenue [15] have discussed $\boldsymbol{A}$-stable one- and two-step numerical integration methods, respectively, which are exponentially fitted at infinity. Both schemes ensure exponential fitting by a suitable choice of a 
free parameter. This approach was further extended to construct a stiffly stable $k$ step method of order $k+2$ in Enright [7].

We shall construct from both Eqs. (1.6) and (1.7) explicit one-step numerical integration formulas of fixed order four which possess adequate stability and convergent characteristics to cope with both stiff and highly oscillatory systems of ordinary differential equations. For linear systems the interpolating functions are global in the sense that the stiffness/oscillatory matrices have constant entries which are determined by solving a set of linear equations at the first step of the integration procedure. The implicit scheme proposed in Fatunla [11] is further developed using the interpolating function (1.7). The need to solve nonlinear equations for the stiffness/oscillatory parameters is eliminated.

2. Development of the Integration Formulas. Let $y_{n+j}$ denote the numerical estimate of the theoretical solution $y(x)$ at $x=x_{n+j}$, and adopt the notation $f_{n+j}=$ $f\left(x_{n+j}, y_{n+j}\right)$.

By demanding that the interpolating function (1.6) coincide with the theoretical solution at the endpoints of the interval $\left[x_{n}, x_{n+1}\right]$, the following pair of equations are readily obtainable:

$$
y_{n}=\left(I-e^{\Omega_{1} x_{n}}\right) A-\left(I-e^{-\Omega_{2} x_{n}}\right) B+C,
$$

and

$$
y_{n+1}=\left(I-e^{\Omega_{1} x_{n+1}}\right) A-\left(I-e^{-\Omega_{2} x_{n}+1}\right) B+C .
$$

If the interpolating function satisfies the differential equation (1.5) at $x=x_{n}$, we have the relationship

$$
f_{n}=-\Omega_{1} e^{\Omega_{1} x_{n}} A-\Omega_{2} e^{-\Omega_{2} x_{n}} B
$$

and

$$
f_{n}^{(1)}=-\Omega_{1}^{2} e^{\Omega_{1} x_{n}} A+\Omega_{2}^{2} e^{-\Omega_{2} x_{n}} B
$$

We now solve Eqs. (2.3) and (2.4) to give

$$
e^{\Omega_{1} x_{n}} A=-\frac{f_{n}^{(1)}+\Omega_{2} f_{n}}{\Omega_{1}\left(\Omega_{1}+\Omega_{2}\right)},
$$

and

$$
e^{-\Omega_{2} x_{n}} B=\frac{f_{n}^{(1)}-\Omega_{1} f_{n}}{\Omega_{2}\left(\Omega_{1}+\Omega_{2}\right)} .
$$

By subtracting Eq. (2.1) from (2.2) and adopting (2.5)-(2.6) (noting that $\left.x_{n+1}=x_{n}+h\right)$ yields the integration formula

$$
y_{n+1}=y_{n}+R f_{n}+S f_{n}^{(1)}
$$


where the matrices $R$ and $S$ are defined as

$$
\begin{gathered}
R=\Omega_{2} \Phi-\Omega_{1} \Psi, \\
S=\Phi+\Psi ;
\end{gathered}
$$

and the diagonal matrices $\Phi, \Psi$, respectively, have entries

$$
i_{\Phi}=\frac{e^{i} \Omega_{1} h}{i \Omega_{1}\left({ }^{i} \Omega_{1}+i \Omega_{2}\right)}
$$

and

$$
{ }^{i} \Psi=\frac{e^{-i \Omega_{2} h}-1}{{ }^{i} \Omega_{2}\left({ }^{i} \Omega_{1}+{ }^{i} \Omega_{2}\right)},
$$

for $i=1(1) m$.

In the event that a component of the stiffness/oscillatory matrix ( ${ }^{i} \Omega_{1}$ say) does vanish, by L'Hospital's rule, the corresponding component of $\Phi$ specified by (2.10) is obtained as

$$
{ }^{j} \Phi={ }^{j} \Omega_{2}^{-1} h,
$$

and the resultant integration formula is given by

$$
{ }^{j} y_{n+1}={ }^{j} y_{n}+h^{j} f_{n}+\left({ }^{j} \Omega_{2}^{-1} h+{ }^{j} \Psi\right)^{j} f_{n}^{(1)} .
$$

We now discuss the case of the complex interpolating function (1.7). The components of the oscillatory/stiffness matrices $\Omega_{1}$ and $\Omega_{1}^{*}$ are, respectively, given as follows:

$$
{ }^{j} \Omega_{1}=\lambda+i u
$$

and

$$
{ }^{i} \Omega_{1}^{*}=\lambda-i u,
$$

for some real numbers $\lambda, u$.

By imposing the same constraints on (1.7) as on (1.6), we still obtain the integration formula (2.7) but now with the components ${ }^{i} R,{ }^{i} S$ of $R$ and $S$, respectively, given by

$$
{ }^{j} R(\lambda, u)=\frac{e^{\lambda h}\left[\left(\lambda^{2}-u^{2}\right) \sin (h u)-2 \lambda u \cos (h u)\right]+2 \lambda u}{u\left(\lambda^{2}+u^{2}\right)},
$$

-and

$$
{ }^{i} S(\lambda, u)=\frac{e^{\lambda h}[\lambda \sin (h u)-u \cos (h u)]+u}{u\left(\lambda^{2}+u^{2}\right)} .
$$

When both $\lambda$ and $u$ are simultaneously close to zero, Eqs. (2.16) and (2.17) both reduce to

$$
{ }^{j} R(\lambda, u)=h
$$


and

$$
{ }^{j} S(\lambda, u)=\frac{h^{2}}{2}
$$

respectively, the resultant integration formula thus reducing to the popular Taylor series method of order two.

3. Evaluation of Stiffness/Oscillatory Parameters. The Taylor expansion of $y_{n+1} \equiv y\left(x_{n}+h\right)$ about $x=x_{n}$ is given by

$$
y_{n+1} \equiv \sum_{r=0}^{\infty} \frac{h^{r} y^{r}\left(x_{n}\right)}{r !},
$$

while the Maclaurin series of $e^{\Omega_{1} h}$ and $e^{-\Omega_{2} h}$ can be, respectively, given by

$$
\begin{gathered}
e^{\Omega_{1} h}=\sum_{r=0}^{\infty} \frac{\left(\Omega_{1} h\right)^{r}}{r !}, \\
e^{-\Omega_{2} h}=\sum_{r=0}^{\infty}(-1)^{r} \frac{\left(\Omega_{2} h\right)^{r}}{r !} .
\end{gathered}
$$

By the application of Eqs. (2.8)-(2.11) and (3.1)-(3.3) in the integration formula (2.7), it is observed that the coefficients of $h^{0}, h^{1}$ and $h^{2}$ vanish identically.

With the view to obtain numerical estimates for the stiffness/oscillatory parameters, we simply allow the coefficients of $h^{3}$ and $h^{4}$ to vanish, thus yielding the following pair of nonlinear equations:

$$
\left(\Omega_{2}-\Omega_{1}\right) f_{n}^{(1)}-\Omega_{1} \Omega_{2} f_{n}=-f_{n}^{(2)},
$$

and

$$
-\left(\Omega_{1}^{2}-\Omega_{1} \Omega_{2}+\Omega_{2}^{2}\right) f_{n}^{(2)}+\Omega_{1} \Omega_{2}\left(\Omega_{2}-\Omega_{1}\right) f_{n}^{(1)}=-f_{n}^{(3)} .
$$

By adopting (3.4) as a definition of $\Omega_{1} \Omega_{2} f_{n}$, Eq. (3.5) becomes

$$
\left(\Omega_{2}-\Omega_{1}\right) f_{n}^{(2)}-\Omega_{1} \Omega_{2} f_{n}^{(1)}=-f_{n}^{(3)} .
$$

If the set of equations (3.4) and (3.6) were to be meaningful, it is desirable that

Let

$$
\operatorname{det}\left(\begin{array}{cc}
{ }^{i} f_{n}^{(2)} & { }^{i} f_{n}^{(1)} \\
{ }^{i} f_{n}^{(1)} & { }^{i} f_{n}
\end{array}\right) \neq 0, \quad i=1(1) m
$$

$$
{ }^{i} D={ }^{i} \Omega_{2}-{ }^{i} \Omega_{1}, \quad i=1(1) m
$$

and

$$
{ }^{i} E={ }^{i} \Omega_{1}{ }^{i} \Omega_{2}, \quad i=1(1) m .
$$

Equations (3.4) and (3.6) can now be expressed as an $m$ pair of linear equations

$$
\begin{aligned}
& { }^{i} D^{i} f_{n}^{(2)}-{ }^{i} E^{i} f_{n}^{(1)}=-{ }^{i} f_{n}^{(3)}, \\
& { }^{i} D f_{n}^{i(1)}-{ }^{i} E{ }^{i} f_{n}=-i f_{n}^{(2)},
\end{aligned}
$$


These $m$ pairs of equations can be readily solved for ${ }^{i} D$ and ${ }^{i} E$ by Cramer's rule to give

$$
{ }^{i} D=\frac{{ }^{i} f_{n}{ }^{i} f_{n}^{(3)}-{ }^{i} f_{n}^{(1) i} f_{n}^{(2)}}{{ }^{i} f_{n}^{(1) i} f_{n}^{(1)}-{ }^{i} f_{n}{ }^{i} f_{n}^{(2)}}, \quad i=1(1) m
$$

and

$$
{ }^{i} E=\frac{{ }^{i} f_{n}^{(1) i} f_{n}^{(3)}-{ }^{i} f_{n}^{(2) i} f_{n}^{(2)}}{{ }^{i} f_{n}^{(1) i} f_{n}^{(1)}-{ }^{i} f_{n}{ }^{i} f_{n}^{(2)}}, \quad i=1(1) m .
$$

The numerical values obtained for ${ }^{i} D$ and ${ }^{i} E$ can be substituted in Eqs. (3.8) and (3.9) to generate the oscillatory/stiffness parameters as

and

$$
{ }^{i} \Omega_{1}=1 / 2\left[-{ }^{i} D+\sqrt{ }\left({ }^{i} D^{2}+4^{i} E\right)\right]
$$

$$
{ }^{i} \Omega_{2}={ }^{i} \Omega_{1}+{ }^{i} D
$$

The complex interpolation formula (1.7) is adopted if in (3.13), the following relationship holds:

$$
{ }^{i} D^{2}<-4^{i} E \text {. }
$$

Equations (3.4) and (3.6) are now replaced by the pair of matrix equations

$$
-\left(\Omega+\Omega^{*}\right) f_{n}^{(1)}+\Omega \Omega^{*} f_{n}=-f_{n}^{(2)}
$$

and

$$
-\left(\Omega+\Omega^{*}\right) f_{n}^{(2)}+\Omega \Omega^{*} f_{n}^{(1)}=-f_{n}^{(3)} .
$$

The matrix $\Omega$ in the case of the linear system $y^{\prime}=A y$ is determined by either

$$
-\left(\Omega+\Omega^{*}\right) A^{2} y_{n}+\Omega \Omega^{*} A y_{n}=-A^{3} y_{n}
$$

or

$$
-\left(\Omega+\Omega^{*}\right) A^{3} y_{n}+\Omega \Omega^{*} A^{2} y_{n}=-A^{4} y_{n} .
$$

In case $A$ is nonsingular, both Eqs. (3.18) and (3.19) reduce to the matrix equation

$$
A^{2}-\left(\Omega+\Omega^{*}\right) A+\Omega \Omega^{*}=0 .
$$

The following theorem guarantees the existence of a solution to (3.20).

THEOREM 1. If $A$ is a real, normal $n \times n$ matrix with distinct eigenvalues $\left(\lambda_{1}, \bar{\lambda}_{1}, \lambda_{2}, \bar{\lambda}_{2}, \ldots, \lambda_{r}, \bar{\lambda}_{r}, \mu_{1}, \mu_{2}, \ldots, \mu_{s}\right)$, where $2 r+s=n$, and if $U$ is a unitary matrix such that $U^{-1} A U=G$ is diagonal, then the solution to matrix equation (3.20) is given by $\Omega=U \mho U^{-1}$ with $\mho=\Sigma_{i=1}^{r} \oplus \lambda_{i} I_{2} \oplus \Sigma_{i=1}^{s} \oplus \mu_{i} I_{1}$, where $I_{r}$ is the $r \times r$ unit matrix.

Proof. Since $A$ is a real matrix, and if $\lambda$ is an eigenvalue of $A$, so is $\bar{\lambda}$. Hence, we can find a unitary matrix $U$ such that $U^{-1} A U=G$, where $G$ is a diagonal matrix with entries $\left(\lambda_{1}, \bar{\lambda}_{1}, \lambda_{2}, \bar{\lambda}_{2}, \ldots, \lambda_{r}, \bar{\lambda}_{r}, \mu_{1}, \mu_{2}, \ldots, \mu_{s}\right)$. Here each $\mu$ is real and $2 r+s=n$. 
If $\mho=U^{-1} \Omega U$, then the matrix equation (3.20) is equivalent to

$$
G^{2}-\left(\mho+\mho^{*}\right) G+\mho \mho^{*}=0
$$

Now a solution of

$$
\left(\begin{array}{ll}
\lambda^{2} & 0 \\
0 & \bar{\lambda}^{2}
\end{array}\right)-(Z+\bar{Z})\left(\begin{array}{ll}
\lambda & 0 \\
0 & \bar{\lambda}
\end{array}\right)+Z \bar{Z} I_{2}=0
$$

is given by $Z=\lambda$, and a solution of

$$
\mu^{2}-(Z+\bar{Z}) \mu+Z \bar{Z} I_{1}=0
$$

is given by $Z=\mu$ ( $\mu$ is real). Hence, a solution of (3.21) is given by

$$
\mho=\sum_{i=1}^{r} \oplus \lambda_{i} I_{2} \oplus \sum_{i=1}^{s} \oplus \mu_{i} I_{1}
$$

giving a solution $\Omega=U \mho U^{-1}$ for Eq. (3.20).

4. Stability Considerations. We now apply the integration formula (2.13) to the scalar test equation

$$
y^{\prime}=\lambda y,
$$

where $\lambda$ is a complex constant with negative real part.

The numerical solution is given as

$$
y_{n+1}=p\left(\lambda, \Omega_{2}, h\right) y_{n},
$$

where the characteristic equation $p\left(\lambda, \Omega_{2}, h\right)$ is given by

$$
p\left(\lambda, \Omega_{2}, h\right)=1+\lambda h+\lambda^{2}\left(\Omega_{2} \dot{h}+e^{-\Omega_{2} h}-1\right) / \Omega_{2}^{2} .
$$

Equation (3.11) gives the following relationship:

$$
\Omega_{2}={ }^{i} D=D \text {. }
$$

For the test problem (4.1) condition (3.7) is violated. However, by setting $\Omega_{1}=0$ in Eq. (3.4), we readily obtain

$$
\Omega_{2}=-\lambda
$$

and from Eq. (3.14), we also have that

$$
D=-f_{n}^{(2)} / f_{n}^{(1)}
$$

These in Eq. (4.3) give

$$
P(\lambda,-\lambda, h)=e^{\lambda h}
$$

We further consider the application of the integration formula (2.7) to test problem (4.1) for the case when the stiffness/oscillatory parameter is imaginary. Thus, $R(\lambda, u)$ and $S(\lambda, u)$ used in the integration formula (2.7) to the test problem

(4.1) are specified by

$$
R(0, u)=\frac{\sin (h u)}{u}
$$


and

$$
S(0, u)=\frac{1-\cos (h u)}{u^{2}}
$$

Let

$$
\lambda=i z, \quad i^{2}=-1
$$

The resultant integration formula is given by

$$
y_{n+1}=q(\lambda, u, h) y_{n},
$$

where the characteristic equation can be obtained as

$$
q(\lambda, u, h)=1+\frac{(1-\cos (h u)) \lambda^{2}}{u^{2}}+\frac{\sin (h u) \lambda}{u} .
$$

With the same argument as in the case of real $\lambda$, we can also obtain

$$
D=-i z=-\lambda=\Omega_{1}^{*} \text {. }
$$

This in (4.11) gives

$$
q(\lambda, u, h)=1-(1-\cos (h u))+i \sin (h u)=e^{i h u}=e^{\lambda h} .
$$

Equations (4.7) and (4.14) together with Definitions 1 and 2, thus establish the $L$-stability and exponential fitting of the proposed integration formulas.

5. Local Truncation Error. We now associate with the integration formula (2.7) the operator $V y(x), h]$ specified as

$$
V(y(x), h)=y(x+h)-y(x)+\left(\Omega_{2} \gamma-\Omega_{1} \sigma\right) f(x, y)-(\gamma+\sigma) f^{(1)}(x, y)
$$

for an arbitrary function $y(x) \in C^{5}(S)$. The local truncation error $T_{n+1}$ at $x=x_{n+1}$ is, hence, given as $\left.V y\left(x_{n}\right), h\right]$, where $y\left(x_{n}\right)$ denotes the solution to the intital value problem (1.5). By using the Taylor expansion of $\eta y(x), h]$ about $x=x_{n}$ with the localizing assumption that there is no previous error (i.e. $\left.y_{n}=y\left(x_{n}\right)\right)$, the truncation error for the integration formula (2.7) with constraints (3.4) and (3.6) can be derived as

$$
\begin{aligned}
T_{n+1}=\frac{h^{5}}{5 !}\left(\Omega_{1}+\Omega_{2}\right)^{-1}\left[\left(\Omega_{1}+\Omega_{2}\right) f_{n}^{(4)}\right. & -\Omega_{1}^{4}\left(f_{n}^{(1)}+\Omega_{2} f_{n}\right) \\
& \left.+\Omega_{2}^{4}\left(f_{n}^{(1)}-\Omega_{1} f_{n}\right)\right]+O\left(h^{6}\right) .
\end{aligned}
$$

The corresponding truncation error for the integration formula (2.13) is given by

$$
T_{n+1}=\frac{h^{5}}{5 !}\left(f_{n}^{(4)}-\Omega_{2}^{3} f_{n}^{(1)}\right)+O\left(h^{6}\right)
$$

while the truncation error when $R(\lambda, \mu)$ and $S(\lambda, u)$ are specified by Eqs. (2.16) and (2.17) is

$$
T_{n+1}=\frac{h^{5}}{5}\left[f_{n}^{(4)}+\left(\lambda^{2}+u^{2}\right)\left(3 \lambda^{2}-u^{2}\right) f_{n}+4 \lambda\left(u^{2} \lambda^{2}\right) f_{n}^{(1)}\right]+O\left(h^{6}\right) .
$$

From Eqs. (5.3) and (5.4) we deduce that all the proposed explicit integration formulas are of fixed order four. 
6. Extension of Nonlinear Scheme. We recall the implicit numerical integration formula proposed in Fatunla [10]

(6.1) $y_{n+1}^{[s+1]}=y_{n+1}^{[s]}-\left[I-\theta J_{n+1}^{[s]}\right]^{-1}\left[y_{n+1}^{[s]}-\theta f_{n+1}^{[s]}-G_{n}\right], \quad s=0,1,2, \ldots$, where $J_{n+1}^{[s]}$ denotes the Jacobian specified as

$$
J_{n+1}^{[s]}=\frac{\delta f}{\delta y}\left(x_{n+1}, y_{n+1}^{[s]}\right), \quad f_{n+1}^{[s]}=f\left(x_{n+1}, y_{n+1}^{[s]}\right),
$$

and

$$
G_{n}=y_{n}-(\gamma+\sigma) f_{n}
$$

The components of $\theta, \gamma, \sigma$ are obtained as

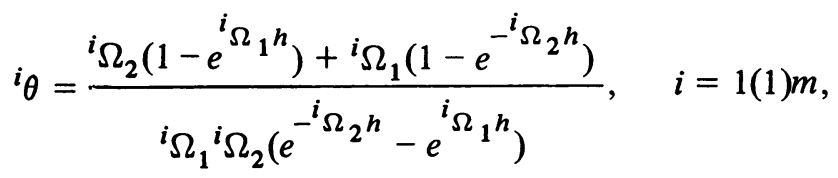

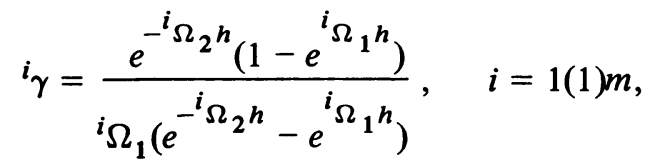

and

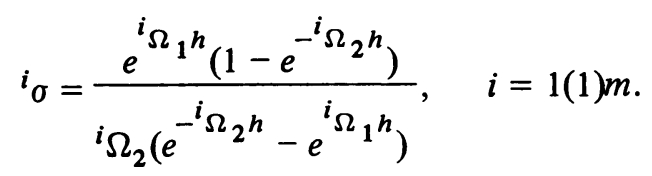

The corresponding truncation error for the integration formula (6.1) was obtained as

$$
\begin{aligned}
T_{n+1}=\frac{h^{6}}{720}\left\{9 f_{n}^{(4)}+\right. & 5\left(\Omega_{2}-\Omega_{1}\right) f_{n}^{(3)}+5\left(\Omega_{1}^{2}-\Omega_{1} \Omega_{2}+\Omega_{2}^{2}\right) f_{n}^{(2)} \\
& -9\left(\Omega_{2}-\Omega_{1}\right)\left(\Omega_{1}^{2}+\Omega_{2}^{2}\right) f_{n}^{(1)} \\
+ & {\left.\left[5 \Omega_{1}^{2} \Omega_{2}^{2}-9 \Omega_{1} \Omega_{2}\left(\Omega_{1}^{2}-\Omega_{1} \Omega_{2}+\Omega_{2}^{2}\right)\right] f_{n}\right\}+O\left(h^{7}\right) }
\end{aligned}
$$

In the case when the oscillatory/stiffness parameters have complex values, the components of $\theta$ and $(\gamma+\delta)$ in Eq. (6.1) are replaced by

$$
\begin{gathered}
{ }^{i} \theta=\frac{e^{\lambda h} u \cos (h u)-\lambda \sin (h u)+u}{e^{\lambda h}\left(\lambda^{2}+u^{2}\right) \sin (h u)}, \quad i=1(1) m, \\
{ }^{i}(\gamma+\delta)=\frac{e u^{\lambda h}-[\lambda \sin (h u)+u \cos (h u)]}{\left(\lambda^{2}+u^{2}\right) \sin (h u)},
\end{gathered}
$$

with the truncation error (6.7) replaced by

$$
\begin{aligned}
T_{n+1}=\frac{h^{6}}{720}\{ & 9 f_{n}^{(4)}-10 \lambda f_{n}^{(3)}+5\left(3 \lambda^{2}-u^{2}\right) f_{n}^{(2)} \\
& \left.+36\left(\lambda^{2}-u^{2}\right) f_{n}^{(1)}+4\left(\lambda^{2}+u^{2}\right)\left(8 \lambda^{2}-u^{2}\right) f_{n}\right\}+O\left(h^{7}\right) .
\end{aligned}
$$




\section{Numerical Examples.}

Example 1. We first consider the linear system

$$
y^{\prime}=\left[\begin{array}{ccc}
-.1 & -49.9 & 0 \\
0 & -50 & 0 \\
0 & 70 & -120
\end{array}\right] y, \quad y(0)=\left[\begin{array}{l}
2 \\
1 \\
2
\end{array}\right]
$$

in the interval $0 \leqslant x \leqslant 15$. The eigenvalues of the Jacobian of (7.1) are $\lambda_{1}=-0.1$, $\lambda_{2}=-50, \lambda_{3}=-120$. The theoretical solution is given as

$$
\begin{aligned}
& { }^{1} y(x)=e^{-0.1 x}+e^{-50 x}, \\
& { }^{2} y(x)=e^{-50 x}, \\
& { }^{3} y(x)=e^{-50 x}+e^{-120 x} .
\end{aligned}
$$

The new explicit scheme performs better than the DIFSUB [13], [14] and the

\begin{tabular}{|c|c|c|c|c|c|c|}
\hline Method & $\begin{array}{c}\text { Max } \\
\text { Order }\end{array}$ & Steps & $\begin{array}{c}\text { Function } \\
\text { Calls }\end{array}$ & $\begin{array}{l}\text { Back } \\
\text { Solves }\end{array}$ & $\begin{array}{c}\text { LU } \\
\text { Decomp }\end{array}$ & $\begin{array}{c}\text { Accurate } \\
\text { Digits }\end{array}$ \\
\hline DIFSUB [13], [14] & 6 & 353 & 1024 & 969 & 18 & 9.3 \\
\hline $\begin{array}{l}\text { BLENDED } \\
\text { DIFSUB [22] }\end{array}$ & 11 & 234 & 595 & 1056 & 22 & 10.4 \\
\hline $\begin{array}{l}\text { EXPLICIT } \\
\text { SCHEME }\end{array}$ & 4 & 75 & 75 & - & - & 12.5 \\
\hline
\end{tabular}
blended DIFSUB [24]. Details of the numerical results are given in Table 7.1.

TABLE 7.1

Numerical results for Example 1

\begin{tabular}{|c|c|c|c|c|c|c|}
\hline$\epsilon$ & $\begin{array}{c}\text { Max } \\
\text { Order }\end{array}$ & Steps & $\begin{array}{l}\text { Func } \\
\text { Eval }\end{array}$ & $\begin{array}{l}\text { Back } \\
\text { Solves }\end{array}$ & $\begin{array}{c}\text { LU } \\
\text { Decomp }\end{array}$ & $\begin{array}{c}\text { Accurate } \\
\text { Digits }\end{array}$ \\
\hline \multicolumn{7}{|c|}{ DIFSUB [13], [14] } \\
\hline $10^{-2}$ & (4) & $(1001)$ & $(3002)$ & $(2959)$ & (7) & (1.1) \\
\hline \multicolumn{7}{|c|}{ "too much work"; integration abandoned at $x=8.3$} \\
\hline \multicolumn{7}{|c|}{ blended DIFSUB } \\
\hline $10^{-10}$ & (12) & $(1001)$ & & $(5580)$ & (21) & $(10.3)$ \\
\hline \multicolumn{7}{|c|}{ "too much work"; integration abandoned at $x=5.1$} \\
\hline \multicolumn{7}{|c|}{ Explicit Scheme } \\
\hline unspec & ified 4 & 200 & 200 & - & - & 14.2 \\
\hline
\end{tabular}

TABLE 7.2

Numerical results for Example 2 
Example 2. We further consider the linear problem

(7.2) $y^{\prime}=\left[\begin{array}{rrrrrr}-10 & 100 & 0 & 0 & 0 & 0 \\ -100 & -10 & 0 & 0 & 0 & 0 \\ 0 & 0 & -4 & 0 & 0 & 0 \\ 0 & 0 & 0 & -1 & 0 & 0 \\ 0 & 0 & 0 & 0 & -.5 & 0 \\ 0 & 0 & 0 & 0 & & -.1\end{array}\right] y, \quad y_{i}(0)=1, \quad i=1(1) 6$

in the range $0 \leqslant t \leqslant 20$. The eigenvalues of the Jacobian are $\lambda_{1,2}=-10 \pm 100 i$, $\lambda_{3}=-4, \lambda_{4}=-1, \lambda_{5}=-.5$, and $\lambda_{6}=-.1$. This problem is particularly, troublesome for both DIFSUB and the blended DIFSUB as can be seen from Table 7.2.

Example 3. We now consider the initial value problem of Liniger and Willoughby [20].

$$
y^{\prime}=\left(\begin{array}{cc}
-2000 & 1000 \\
1 & -1
\end{array}\right) y+\left(\begin{array}{l}
1 \\
0
\end{array}\right), \quad y(0)=\left(\begin{array}{l}
0 \\
0
\end{array}\right)
$$

in the interval $0 \leqslant x \leqslant 5$.

This problem is contained in the test batch recommended by Bjurel et al. [4], and was also considered in Lambert [16]. In addition, it is solved with the blended DIFSUB [24].

TABLE 7.3

Numerical results for Example 3

\begin{tabular}{lllll|llll}
\multicolumn{4}{c}{$10^{4} x^{1} y$} & \multicolumn{4}{c}{$10^{4} x^{2} y$} \\
\hline & $\begin{array}{l}\text { Explicit } \\
\text { Scheme } \\
h=0.5\end{array}$ & $\begin{array}{l}\text { Blended } \\
\text { DIFSUB }[24] \\
\text { Variable } h \\
\epsilon=10^{-6}\end{array}$ & $\begin{array}{l}\text { Lambert } \\
{[16]} \\
h=0.01\end{array}$ & $\begin{array}{l}\text { Theoretical } \\
\text { Solution }\end{array}$ & $\begin{array}{l}\text { Explicit } \\
h=0.5\end{array}$ & $\begin{array}{l}\text { Blended } \\
\text { DIFSUB } \\
{[24]}\end{array}$ & $\begin{array}{l}\text { Lambert } \\
{[16]} \\
h=0.01\end{array}$ & $\begin{array}{l}\text { Theoretical } \\
\text { Solution }\end{array}$ \\
\hline 0.5 & 6.1038 & 6.1038 & 6.0731 & 6.0795 & 2.2096 & 2.2096 & 2.1467 & 2.1574 \\
1.0 & 6.9655 & 6.9650 & 6.9427 & 6.9467 & 3.9324 & 3.9315 & 3.8854 & 3.8922 \\
1.5 & 7.6365 & 7.6362 & 7.6175 & 7.6221 & 5.2743 & 5.2735 & 5.2371 & 5.2433 \\
2.0 & 8.1592 & 8.1590 & 8.1444 & 8.1481 & 6.3194 & 6.3189 & 6.2901 & 6.2955 \\
2.5 & 8.5663 & 8.5662 & 8.5555 & 8.5577 & 7.1333 & 7.1332 & 7.1106 & 7.1149 \\
3.0 & 8.8834 & 8.8833 & 8.8741 & 8.8768 & 7.7673 & 7.7671 & 7.7492 & 7.7531 \\
3.5 & 9.1303 & 9.1303 & 9.1263 & 9.1252 & 8.2610 & 8.2609 & 8.2592 & 8.2500 \\
4.0 & 9.3226 & 9.3226 & 9.3238 & 9.3187 & 8.6456 & 8.6455 & 8.6525 & 8.6371 \\
4.5 & 9.4724 & 9.4724 & 9.4765 & 9.4694 & 8.9452 & 8.9451 & 8.9564 & 8.9386 \\
5.0 & 9.5891 & 9.5891 & 9.5945 & 9.5867 & 9.1784 & 9.1784 & 9.1915 & 9.1734 \\
\hline
\end{tabular}

The eigenvalues of the Jacobian to (7.3a) are $\lambda_{1}=-2000.5$ and $\lambda_{2}=-0.5$, thus yielding a stiffness ratio 4001 . The stiffness matrices which have constant entries were obtained as

$$
\Omega_{1}=\left(\begin{array}{cc}
-0.499875 & 0 \\
0 & -0.499875
\end{array}\right), \quad \Omega_{2}=\left(\begin{array}{cc}
2000.5 & 0 \\
0 & 2000.5
\end{array}\right)
$$


Table (7.3) gives the numerical results for the explicit scheme, Lambert's scheme and the blended DIFSUB. The global relative error for the blended DIFSUB is $0.743400 \mathrm{D}-5$ while the global relative error for the explicit scheme is $0.5746777037 \mathrm{E}-05$. The computational cost and function evaluation on IBM 360/75 for the blended DIFSUB are, respectively, $\$ 1.84$ and 107 while that of the explicit scheme is $\$ 0.77$ and 10 , respectively.

\section{TABLE 7.4}

Numerical results for Example 4

\begin{tabular}{|c|c|c|c|}
\hline \multicolumn{4}{|c|}{ EXPLICIT } \\
\hline $\begin{array}{l}\text { No. of } \\
\text { Fn. Eval. of } \\
f^{(r)}, r=0,1,2,3\end{array}$ & $h$ & $y(1)$ & $y(2)$ \\
\hline 5 & 0.2000 & 1.8716065 & -0.14358810 \\
\hline 7 & 0.1500 & 1.8711045 & -0.14574713 \\
\hline 10 & 0.1000 & 1.8705973 & -0.14610294 \\
\hline 20 & 0.0500 & 1.8694380 & -0.14823599 \\
\hline 40 & 0.0250 & 1.8694389 & -0.14823587 \\
\hline 80 & 0.0125 & 1.8694388 & -0.14823588 \\
\hline \multicolumn{4}{|c|}{ Blended DIFSUB [24] } \\
\hline $\begin{array}{l}\text { No. of } \\
\text { Fn. Eval. }\end{array}$ & $h_{0}=10^{-3}$ & $y(1)$ & $\begin{array}{c}y(2) \\
\end{array}$ \\
\hline 120 & $\xi=10^{-6}$ & 1.86944 & -0.148236 \\
\hline \multicolumn{4}{|c|}{ IMPLICIT [11] } \\
\hline $\begin{array}{l}\text { No. of } \\
\text { Fn. Eval. of } \\
f^{(r)}, r=0,1,2,3\end{array}$ & $h$ & $y(1)$ & $y(2)$ \\
\hline- & 0.2000 & $*$ & $*$ \\
\hline- & 0.1500 & $*$ & $*$ \\
\hline 42 & 0.1000 & 1.8693953 & -0.14824187 \\
\hline 81 & 0.0500 & 1.8694357 & -0.14823631 \\
\hline 161 & 0.0250 & 1.8694387 & -0.14823589 \\
\hline 321 & 0.0125 & 1.8694389 & -0.14823587 \\
\hline *No convergence & & & \\
\hline
\end{tabular}

The global errors were computed as

$$
\bar{e}=\max _{0<t<N} \sum_{i=1}^{m}\left[\left(\frac{i y_{t}-i y\left(x_{t}\right)}{i \omega}\right)^{2}\right]^{1 / 2},
$$

$$
{ }^{i} \omega=\max _{1<i<m}\left\{1,{ }^{i} y_{0},{ }^{i} y_{1}, \ldots,{ }^{i} y_{N}\right\}
$$


Example 4. Van der Pol's oscillator (considered in Enright et al.

$$
\begin{aligned}
& y_{1}^{\prime}=y_{2}, \quad y_{1}(0)=2, \\
& y_{2}^{\prime}=5\left(1-y_{1}^{2}\right) y_{2}-y_{1}, \quad y_{2}(0)=0, \quad 0 \leqslant x \leqslant 1 \text {. }
\end{aligned}
$$

Eigenvalues: -0.067 and $-15 \rightarrow 5.7$ and $-1.5 \rightarrow 3.6$ and $1.4 \rightarrow 2.4 \pm 2.8 i \rightarrow$ $-0.052 \pm 8.8 i \rightarrow 2.0 \rightarrow 9.5 i \rightarrow-5.9 \rightarrow 4.5 i \rightarrow-2.0$ and $-12 \rightarrow 0.050$ and $-15 \rightarrow 1.1$ and -3.4 .

The numerical computation was effected with both the explicit and the implicit schemes using uniform mesh-sizes $h=0.2,0.15,0.1,0.05,0.025$ and 0.0125 . The same problem was solved with the blended DIFSUB using an initial mesh-size $h=$ $10^{-3}$ as suggested in Enright et al. [8].

TABLE 7.5

Numerical results for Example 5

\begin{tabular}{rcc}
\hline \multicolumn{3}{c}{$x_{f}=10 \pi$ uniform $h=\pi / 20$} \\
$x$ & $10^{12} \times{ }^{1} T_{t+1}$ & $10^{12} \times{ }^{2} T_{t+1}$ \\
$\pi$ & 0.16918 & 0.00949 \\
$2 \pi$ & 0.02447 & 0.02233 \\
$3 \pi$ & 0.29342 & 0.03504 \\
$4 \pi$ & 0.61126 & 0.04402 \\
$5 \pi$ & 0.92906 & 0.05704 \\
$6 \pi$ & 1.2468 & 0.07060 \\
$7 \pi$ & 1.56451 & 0.08478 \\
$8 \pi$ & 0.09729 & 0.09991 \\
$9 \pi$ & 1.29056 & 1.06429 \\
$10 \pi$ & 1.60815 & 0.12150
\end{tabular}

One evaluation of oscillatory/stiffness parameters.

From Table 7.4 the new schemes compare favorably with the blended DIFSUB.

Example 5.

$$
y^{\prime}=\left(\begin{array}{cc}
-10^{-5} & 100 \\
-100 & -10^{-5}
\end{array}\right) y, \quad y(0)=\left(\begin{array}{l}
0 \\
1
\end{array}\right), \quad 0 \leqslant x \leqslant 10 \pi .
$$

This is the model problem (1.2) with $\epsilon=10^{-5}$ and $\omega=100$ whose theoretical solution is

$$
y(x)=e^{-10^{-5} x}\left(\begin{array}{c}
\sin 100 x \\
\cos 100 x
\end{array}\right)
$$

The numerical results in Table (7.5) are obtained with a uniform mesh-size $h=$ $\pi / 20$, and only one evaluation of the stiffness/oscillatory parameters was obtained and given by

$$
\Omega_{1}=\left(\begin{array}{cc}
-10^{-5}-100 i & 0 \\
0 & -10^{-5}-100 i
\end{array}\right), \quad \Omega_{2}=\left(\begin{array}{cc}
-10^{-5}+100 i & 0 \\
0 & -10^{-5}+100 i
\end{array}\right)
$$


Example 6. We finally consider the nearly periodic initial value problem which was earlier studied by Stiefel-Bettis [26] and Lambert-Watson [17]

$$
y^{\prime \prime}+y^{\prime}=0.001 e^{i x}, y(0)=1, \quad y^{\prime}(0)=0.9995 i, \quad y \in R^{1},
$$

whose theoretical solution is

$$
\left\{\begin{array}{l}
y(x)=u(x)+i v(x), \quad u, v \in R^{1}, \\
u(x)=\cos x+0.0005 x \sin x \\
v(x)=\sin x-0.0005 x \cos x
\end{array}\right.
$$

Equations (7.6b) represent motion on a perturbation of a circular orbit in the complex plane in which the point $y(x)$ spirals slowly outwards such that its distance from the origin at any time $x$ is given as

$$
\tau(x)=\sqrt{ }\left(u^{2}(x)+v^{2}(x)\right) .
$$

The initial value problem (7.6a) can be expressed as

$$
\left\{\begin{array}{l}
{ }^{1} y^{\prime}={ }^{2} y,{ }^{1} y(0)=1 \\
{ }^{2} y^{\prime}=-{ }^{1} y+0.001 \cos x, \quad{ }^{2} y(0)=0 \\
{ }^{3} y^{\prime}={ }^{4} y, \quad{ }^{3} y(0)=0 \\
{ }^{4} y^{\prime}=-{ }^{3} y+0.001 \sin x,
\end{array}\right.
$$

The system (7.6d) was solved with the explicit formulas in the range $0 \leqslant x \leqslant$ $40 \pi$ which corresponds ta 20 orbits of the point $y(x)$. The integration was performed using uniform mesh-sized $h=\pi / 4, \pi / 5, \pi / 6, \pi / 9$, and $\pi / 12$. Two sets of numerical results were generated: In the first set, the oscillatory/stiffness parameters are obtained once at the first step of integration, while in the second set, the oscillatory parameters are evaluated at every step of integration.

The same problem was solved with the symmetric multistep method of Lambert and Watson [17] as well as the Störmer-Cowell five-step multistep formula [24] (both of order 6)

$$
y_{n+5}-2 y_{n+4}+y_{n+3}
$$

$$
=\frac{h}{240}\left(18 f_{n+5}+209 f_{n+4}+4 f_{n+3}+14 f_{n+2}-6 f_{n+1}+f_{n}\right) .
$$

The exact distance from the origin $\tau(x)$ is given by (7.6c), and the approximate distance is $\tau=\sqrt{ }\left({ }^{1} y^{2}+{ }^{3} y^{2}\right)$ at $x=40 \pi$. All the solutions generated by the new scheme spiral outward in agreement with the theoretical solution as well as Lambert's scheme, whilst the first three values generated by Störmer-Cowell scheme spiral inward.

From the Tables (7.6a, b and c) which, respectively, show $\tau,|\tau(x)-\tau|$ and $|\hat{y}(x)-\hat{y}|=\sqrt{ }\left[\left({ }^{1} y(x)-{ }^{1} y\right)^{2}+\left({ }^{3} y(x)-{ }^{3} y\right)^{2}\right]$, we see that despite the fact that the new scheme is of lower order, yet it is more accurate than both the symmetric multistep method as well as the fifth order Störmer-Cowell multistep scheme. 
TABLE 7.6a

\begin{tabular}{lcccc}
\multicolumn{5}{c}{$x_{f}=40 \pi, \tau\left(x_{f}\right)=1.001972$} \\
\hline$h$ & Störmer-Cowell & Symmetric & \multicolumn{2}{c}{ Explicit } \\
\hline & & & $\begin{array}{l}\text { one evaluation } \\
\text { of parameters }\end{array}$ & $\begin{array}{c}\text { repeated eval. } \\
\text { of parameters }\end{array}$ \\
\hline$\pi / 4$ & 0.965645 & 1.003067 & 1.002311 & 1.001972 \\
$\pi / 5$ & 0.993734 & 1.002217 & 1.002205 & 1.001972 \\
$\pi / 6$ & 0.999596 & 1.002047 & 1.002140 & 1.001972 \\
$\pi / 9$ & 1.001829 & 1.001978 & 1.002050 & 1.001972 \\
$\pi / 12$ & 1.001953 & 1.001973 & 1.002016 & 1.001972 \\
\hline
\end{tabular}

TABLE $7.6 \mathrm{~b}$

\begin{tabular}{|c|c|c|c|c|}
\hline \multicolumn{5}{|c|}{$x_{f}=40 \pi, \tau\left(x_{f}\right)=1.001972$} \\
\hline \multirow[t]{3}{*}{$h$} & \multicolumn{2}{|c|}{$10^{6} x|\tau(x)-\tau|$} & & $10^{9} x|\tau(x)-\tau|$ \\
\hline & \multirow[t]{2}{*}{ Störmer-Cowell } & \multirow[t]{2}{*}{ Symmetric } & \multicolumn{2}{|c|}{ Explicit } \\
\hline & & & $\begin{array}{l}\text { one evaluation } \\
\text { of parameters }\end{array}$ & $\begin{array}{l}\text { repeated eval. } \\
\text { of parameters }\end{array}$ \\
\hline$\pi / 4$ & 36327 & 1095 & 339 & 204 \\
\hline$\pi / 5$ & 8238 & 245 & 233 & 66 \\
\hline$\pi / 6$ & 2376 & 75 & 167 & 26 \\
\hline$\pi / 9$ & 143 & 6 & 78 & 3 \\
\hline$\pi / 12$ & 19 & 1 & 44 & 0 \\
\hline
\end{tabular}

TABLE $7.6 \mathrm{c}$

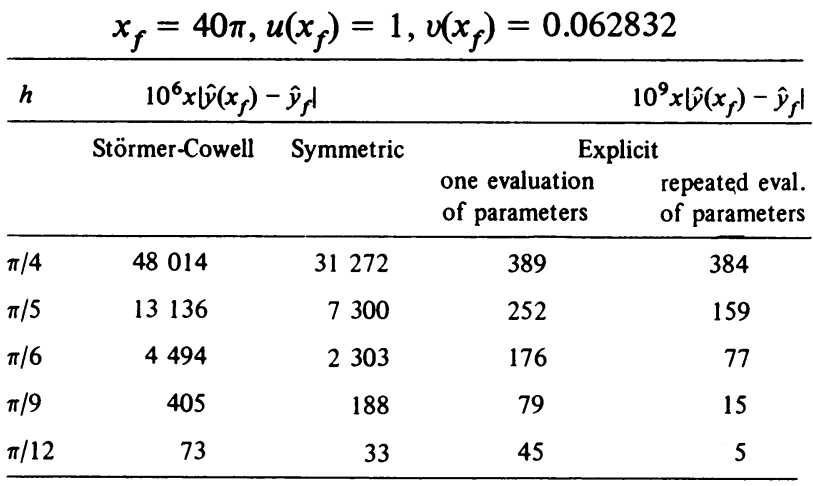

8. Concluding Remarks. The proposed explicit scheme (2.7) and (2.13) is considered to be more efficient and accurate than the DIFSUB and the blended DIFSUB for linear stiff systems of ordinary differential equations. It is equally efficient for highly oscillatory systems as it is capable of admitting fairly large mesh-size and still maintains high degree of accuracy. The major drawback is the need to generate higher-order derivatives, but automatic generation of higher-order derivatives is practicable for an extensive range of problems. The proposed scheme being one-step readily 
accepts the use of variable mesh-size in the event that the asymptotic local error obtained in Section 5 is greater in magnitude than a specified tolerance.

Acknowledgment. The author is indebted to Professor C. W. Gear for his invaluable suggestions, criticism and advice. He went out of his way to ensure that I was comfortable during my short visit at the Department of Computer Science.

Department of Mathematics

University of Benin

Benin City, Nigeria

1. V. AMDURSKY \& A. ZIV, On Numerical Treatment of Stiff, Highly Oscillatory Systems, IBM Technical Report 015, IBM Israel Scientific Center, 1974.

2. V. AMDURSKY \& A. ZIV, On the Numerical Solution of Stiff Linear Systems of the Oscillatory Type, IBM Technical Report 032, IBM Israel Scientific Center, 1975.

3. V. AMDURSKY \& A. ZIV, The Numerical Treatment of Linear Highly Oscillatory ODE Systems by Reduction to Nonoscillatory Types, IBM Technical Report 039, IBM Israel Scientific Center, 1976.

4. G. BJUREL, G. DAHLQUIST, B. LINDBERG, S. LINDEN \& L. ODEN, Survey of Stiff Ordinary Differential Equations, Computer Science Report NA 70.11, Royal Institute of Technology, Stockholm, Sweden, 1970.

5. J. C. BUTCHER, "Implicit Runge-Kutta processes," Math. Comp., v. 18, 1964, pp. 5064.

6. G. DAHLQUIST, “A special stability problem for linear multistep methods,” $B I T, \mathrm{v} .3$, 1963, pp. 27-43.

7. W. H. ENRIGHT, "Second derivative multistep methods for stiff ordinary differential equations," SIAM J. Numer. Anal., v. 11 (2) 1974, pp. 321-331.

8. W. H. ENRIGHT, T. E. HULL \& B. LINDBERG, "Comparing numerical methods for stiff systems of ODEs," $B I T$, v. 15, 1975, pp. 10-48.

9. S. O. FATUNLA, "A new algorithm for numerical solutions of ODEs," Comput. Math. Appl., v. 2, 1976, pp. 247-253.

10. S. O. FATUNLA, "A variable order one step scheme for numerical solution of ODEs," Comput. Math. Appl., v. 4, 1978, pp. 33-41.

11. S. O. FATUNLA, "An implicit two-point numerical integration formula for linear and nonlinear stiff systems of ordinary differential equations," Math. Comp., v. 32, 1978, pp. 1-11.

12 W. GAUTSCHI, "Numerical integration of ODEs based on trigonometric polynomials," Numer. Math., v. 3, 1961, pp. 381-397.

13. C. W. GEAR, "The automatic integration of stiff ordinary differential equations," Proc. IFIP Congress, vol. 1, North-Holland, Amsterdam, 1968, pp. 187-194.

14. C. W. GEAR, "Algorithm 407: DIFSUB for solution of ordinary differential equations," Comm. $A C M$, v. 14, 1971 , pp. 185-190.

15. L. W. JACKSON \& S. K. KENUE, "A fourth order exponentially fitted method," SIAM J. Numer. Anal., v. 11, 1974, pp. 965-978.

16. J. D. LAMBERT, "Nonlinear methods for stiff systems of ordinary differential equations," Proc. Conference on Numerical Solution of Ordinary Differential Equations 363, University of Dundee, 1973, pp. 75-88.

17. J. D. LAMBERT \& I. A. WATSON, "Symmetric multistep methods for periodic initial value problems," J. Inst. Math. Appl., v. 18, 1976, pp. 189-202.

18. J. D. LAWSON, "Generalized Runge Kutta processes for stable systems with large Lipschitz constants," SIAM J. Numer. Anal., v. 4, 1967, pp. 372-380.

19. B. LINDBERG, "On smoothing and extrapolation for the trapezoidal rule," $B I T$, v. 11 , 1971 , pp. 29-52.

20. W. LINIGER \& R. A. WILLOUGHBY, Efficient Numerical Integration of Stiff Systems of Ordinary Differential Equations, IBM Research Report RC 1970, IBM, Yorktown Heights, New York, 1969.

21. W. L. MIRANKER \& G. WAHBA,“An averaging method for the stiff highly oscillatory problems," Math. Comp., v. 30, 1976, pp. 383-399. 
22. W. L. MIRANKER, M. VAN VELDHUIZEN \& G. WAHBA, "Two methods for the stiff highly oscillatory problem," Proc. Numerical Analysis Conference held in Dublin (J. Miller, Ed.), 1976, pp. 257-273.

23. W. L. MIRANKER \& M. VAN VELDHUIZEN, The Method of Envelopes, IBM Research Report RC 6391 (\#27537), Mathematics Division, IBM, Yorktown Heights, New York, 1977.

24. R. D. SKEEL \& A. K. KONG, Blended Linear Multistep Methods, UIUCDCS-R-76-800, Dept. of Comput. Sci., Univ. of Illinois, Urb ana, Ill., 1976.

25. A. D. SNIDER \& G. L. FLEMMING, "Approximation by aliasing with application to "Certaine" stiff differential equations," Math. Comp., v. 28, 1974, pp. 465-473.

26. E. STIEFEL \& D. G. BETTIS, "Stabilization of Cowell's method," Numer. Math., v. 13 , 1969, pp. 154-175. 